Georgian Mathematical Journal

$\mathbf{1}(94)$, No. 4, 419-427

\title{
ON THE INITIAL VALUE PROBLEM FOR FUNCTIONAL DIFFERENTIAL SYSTEMS
}

\author{
V. ŠEDA AND J. ELIA ̌
}

\begin{abstract}
For a system of functional differential equations of an arbitrary order the conditions are established for the initial value problem to be solvable on an infinite interval, and the structure of the set of solutions to this problem is studied.
\end{abstract}

\section{INTRODUCTION}

For the $p$-th order functional differential system

$$
x^{(p)}(t)=f\left(t, x_{t}, \ldots, x_{t}^{(p-1)}\right), \quad b \leq t<+\infty,
$$

we consider the initial value problem

$$
x_{b}^{(k)}=\psi^{(k)} \quad(k=0, \ldots, p-1) .
$$

The invesigation is based on Kubáček's theorem [2] asserting that under certain conditions the set of all fixed points of the compact map in the Fréchet space is a compact $R_{\delta}$-set. It is shown that some restrictions on the growth of the right-hand side of the functional differential system imply that the set of all solutions of the initial value problem for that system is a compact $R_{\delta}$-set in the Fréchet space of $C^{p-1}$-functions. The result extends a similar theorem for first-order functional differential systems proved in [2] and the theorem for second-order functional differential systems proved in [3].

In the sequel we shall use the following notations and assumptions:

Let $h>0, b \in R, d \in N$, and let $|\cdot|$ be a norm in $R^{d}$. Further, let $H_{l}=C^{l}\left([-h, 0], R^{d}\right)$ be provided with the norm

$$
\|x\|_{l}=\max \left\{\sum_{k=0}^{l}\left|x^{(k)}(s)\right|:-h \leq s \leq 0\right\}
$$

1991 Mathematics Subject Classification. 34K05, 34K25. 
for each $x \in H_{l}$ and $l=0, \ldots, p-1$. For brevity $\|\cdot\|_{p-1}$ will be denoted by $\|\cdot\|$.

Let $X=C^{p-1}\left([b, \infty), R^{d}\right)$ be equipped with a topology of locally uniform convergence of the functions and of their $p-1$ derivatives on $[b, \infty)$. In the Fréchet space $X$ the topology is given by the metric

$$
d(x, y)=\sum_{m=1}^{\infty} \frac{1}{2^{m}} \frac{p_{m}(x-y)}{1+p_{m}(x-y)},
$$

where

$$
p_{m}(x)=\sup \left\{\sum_{k=0}^{p-1}\left|x^{(k)}(t)\right|: b \leq t \leq b+m\right\}, \quad x, y \in X, m \geq 1 .
$$

Let $X^{*}=C^{p-1}\left([b-h, \infty), R^{d}\right)$ be a Fréchet space whose topology is determined by seminorms

$$
p_{m}^{*}(x)=\sup \left\{\sum_{k=0}^{p-1}\left|x^{(k)}(t)\right|: b-h \leq t \leq b+m\right\}, x \in X^{*}, m \geq 1 .
$$

For $x \in C\left([b-h, \infty), R^{d}\right)$ we shall denote by $x_{t} \in H_{0}$ the function $x_{t}(s)=x(t+s), s \in[-h, 0], t \geq b$. Clearly $\left(x_{t}\right)^{(k)}(s)=\left(x^{(k)}\right)_{t}(s), s \in$ $[-h, 0], k=0, \ldots, p-1$, and $x \in X^{*}, t \geq b$.

It is assumed throughout the paper that $f \in X\left([b, \infty) \times H_{p-1} \times \cdots \times\right.$ $\left.H_{0}, R^{d}\right), \psi \in H_{p-1}$.

A solution $x$ of (1), (2) is a function $x \in X^{*}$ such that $\left.x\right|_{[b, \infty)} \in$ $C^{p}\left([b, \infty), R^{d}\right)$ abd $x$ satisfies (2) and the functional differential system (1) at each point $t \geq b$.

\section{$\S 1$. Auxiliary Propositions}

Now Kubáček's theorem in [2] will be stated as Lemma 1. In that lemma the compact $R_{\delta}$-set in the metric space $(E, \rho)$ means a nonempty subset $F$ of $E$ which is homeomorphic to the intersection of a decreasing sequence of compact absolute retracts. By [1], p. 92, a metric space $G$ is called an absolute retract when each continuous map $f: K \rightarrow G$ has a continuous extension $g: H \rightarrow G$ for each metric space $H$ and each closed $K \subset H$. For example, a nonempty convex subset of the Fréchet space is an absolute retract.

Lemma 1. Let $M$ be a nonempty closed set in the Fréchet space $(E, \rho)$, $T: M \rightarrow E$ a compact map (i.e., $T$ is continuous and $T(M)$ is a relatively compac set). Denote by $S$ the map $I-T$ where $I$ is the identity map on $E$. Let there exist a sequence $\left\{U_{n}\right\}$ of closed convex sets in $E$ fulfilling the conditions 
(i) $0 \in U_{n}$ for each $n \in N$;

(ii) $\lim _{n \rightarrow \infty} \operatorname{diam} U_{n}=0$,

and a sequence $\left\{T_{n}\right\}$ of maps $T_{n}: M \rightarrow E$ fulfilling the conditions

(iii) $T(x)-T_{n}(x) \in U_{n}$ for each $x \in M$ and each $n \in N$; $U_{n}$.

(iv) the map $S_{n}=I-T_{n}$ is a homeomorphism of the set $S_{n}^{-1}\left(U_{n}\right)$ onto

Then the set $F$ of all fixed points of the map Tis a compact $R_{\delta}$-set.

In the special case $E=X, \rho=d$ Lemma 1 implies

Lemma 2. Let $(X, d)$ be the Fréchet space given above; let $\varphi, \varphi_{n} \in$ $C([b, \infty),[0, \infty))$, and let the following conditions be fulfilled:

(v) For each $t \in[b, \infty)$ the sequence $\left\{\varphi_{n}(t)\right\}$ is nonincreasing and $\lim _{n \rightarrow \infty} \varphi_{n}(t)=0$. Let $r_{k} \in R^{d}, k=0, \ldots, p-1$ and let

$$
M=\left\{x \in X: \sum_{k=0}^{p-1}\left|x^{(k)}(t)-r_{k}\right| \leq \varphi(t), t \geq b, x^{(k)}(b)=r_{k}, k=0, \ldots, p-1\right\} .
$$

It is assumed that $T: M \rightarrow X$ is a compact map with the property $(T(x))^{(k)}(b)=r_{k}, k=0, \ldots, p-1$ for each $x \in M$ and there exists a sequence $\left\{T_{n}\right\}$ of compact maps $T_{n}: M \rightarrow X$ such that $\left(T_{n}(x)^{(k)}(b)=r_{k}\right.$, $k=0, \ldots, p-1$ for each $x \in M$ and

(vi) $\sum_{k=0}^{p-1}\left|\left(T_{n}(x)\right)^{(k)}(t)-(T(x))^{(k)}(t)\right| \leq \varphi_{n}(t), x \in M, t \geq b$;

(vii) for each $n \in N$ there exists a function $\varphi_{* n} \in C([b, \infty),[0, \infty))$ such that $\varphi_{* n}+\varphi_{n} \leq \varphi$ on $[b, \infty)$ and

$$
\sum_{k=0}^{p-1}\left|\left(T_{n}(x)\right)^{(k)}(t)-r_{k}\right| \leq \varphi_{* n}(t), \quad x \in M, \quad t \geq b ;
$$

(viii) the map $S_{n}=I-T_{n}$ is injective on $M$, where $I$ is the identity on $X$.

Then the set $F$ of all fixed points of the map $T$ is a compact $R_{\delta}$.

Proof. The set

$$
U_{n}=\left\{x \in X: \sum_{k=0}^{p-1}\left|x^{(k)}(t)\right| \leq \varphi_{n}(t), t \geq b, x^{(k)}(b)=0, k=0, \ldots, p-1\right\}
$$

is convex and closed on $X$ for each $n \in N$. We shall show that the sequence $\left\{U_{n}\right\}$ satisfies all conditions of Kubáček's theorem when $E=X, \rho=d$ and thus Lemma 2 will follow from Lemma 1.

Clearly, (i) is fulfilled. As to the condition (ii), we choose an arbitrary $\varepsilon>0$. Then there is an $m_{0} \in N$ such that $\sum_{m=m_{[}+1}^{\infty}\left(1 / 2^{m}\right)<\varepsilon / 2$. The 
condition (v) and the Dini theorem imply that the sequence $\left\{\varphi_{n}\right\}$ converges on $[b, \infty)$ locally uniformly to 0 . Therefore for $\varepsilon>0$ and $m_{0} \in N$ there is an $n_{0} \in N$ such that $g_{m}\left(\varphi_{n}\right)=\sup \left\{\left|\varphi_{n}(t)\right|: b \leq t \leq b+m\right\} \leq \varepsilon / 4 m_{0}$ for $n \geq n_{0}$ and $m=1,2, \ldots, m_{0}$. Hence for $n \geq n_{0}$ and $x, y \in U_{n}$ we have

$$
\begin{aligned}
d(x, y) & =\sum_{m=1}^{\infty} \frac{1}{2^{m}} \frac{p_{m}(x-y)}{1+p_{m}(x-y)} \leq \sum_{m=1}^{m_{0}} p_{m}(x-y)+\sum_{m=m_{0}+1}^{\infty} \frac{1}{2^{m}} \leq \\
& \leq \sum_{m=1}^{m_{0}} 2 g_{m}\left(\varphi_{n}\right)+\sum_{m=m_{0}+1}^{\infty} \frac{1}{2^{m}} \leq 2 m_{0} \frac{\varepsilon}{4 m_{0}}+\frac{\varepsilon}{2}=\varepsilon .
\end{aligned}
$$

This implies that (ii) is satisfied. The assumption (iii) follows from (vi) and from the definition of $T, T_{n}, n \in N$.

Now we show the inclusion $U_{n} \subset S_{n}(M)$. The condition (viii) then implies that $S_{n}$ is the bijection of $S_{n}^{-1}\left(U_{n}\right)$ onto $U_{n}$ and is fulfilled, since the continuity of $\left.S_{n}^{-1}\right|_{U_{n}}$ is the consequence of the compactness of $T_{n}$.

Thus we have to prove that for each $y \in U_{n}$ there is an $x_{y} \in M$ such that $x_{y}-T_{n}\left(x_{y}\right)=y$. This means that the map $P_{n}(x)=y+T_{n}(x)$ has a fixed point for each $y \in U_{n}$. The condition (vii) implies

$$
\begin{gathered}
\sum_{k=0}^{p-1}\left|y^{(k)}(t)+\left(T_{n}(x)\right)^{(k)}(t)-r_{k}\right| \leq \sum_{k=0}^{p-1}\left|y^{(k)}(t)\right|+ \\
+\sum_{k=0}^{p-1}\left|\left(T_{n}(x)\right)^{(k)}(t)-r_{k}\right| \leq \varphi_{n}(t)+\varphi_{* n}(t) \leq \varphi(t), \quad t \geq b,
\end{gathered}
$$

and $\left(P_{n}(t)\right)^{(k)}(b)=r_{k}, k=0, \ldots, p-1$, for each $x \in M$. Therefore $P_{n}(M) \subset$ $M$. As $M$ is a closed convex bounded set and $P_{n}(M) \subset M$ is a compact map, by Tikhonov's fixed point theorem, $P_{n}$ has a fixed point.

Now the function $\varphi$ in Lemma 2 will be given as a solution of an integral equation. The existence and some properties of the solution to that equation will be discussed in the following two lemmas.

Lemma 3. Let $\psi \in H_{p-1}, \omega \in C([b, \infty),[0, \infty)), g \in C([0, \infty),[0, \infty))$ be a nondecreasing function. Further, let

$$
\begin{gathered}
\sigma(t) \equiv 0, \quad b \leq t<\infty, \quad \text { if } p=1, \quad \text { and } \\
\sigma(t)=\sum_{l=0}^{p-2}\left(\sum_{k=l+1}^{p-1} \frac{\left|\psi^{(k)}(0)\right|}{(k-l) !}(t-b)^{k-l}, \quad b \leq t<\infty, \quad \text { if } p \geq 2,\right. \\
K(t, s)=\sum_{l=0}^{p-1} \frac{(t-s)^{p-1-l}}{(p-1-l) !}, \quad b \leq s \leq t .
\end{gathered}
$$

Then the following statements hold: 
1. A solution $\varphi \in C([b, \infty),[0, \infty))$ of the integral equation

$$
\varphi(t)=\sigma(t)+\int_{b}^{t} K(t, s) \omega(s) g(\|\psi\|+\varphi(s)) d s, \quad b \leq t<\infty,
$$

exists and satisfies

$$
0 \leq \varphi(t) \leq \lambda(t), \quad b \leq t<\infty
$$

if and only if there exists a function $\lambda \in C([b, \infty),[0, \infty))$ such that

$$
\lambda(t) \geq \sigma(t)+\int_{b}^{t} K(t, s) \omega(s) g(\|\psi\|+\lambda(s)) d s, \quad b \leq t<\infty,
$$

i.e., if and only if there exists an upper solution $\lambda$ of (5).

2. A solution $\varphi$ of (5) (whenever it exists) is a nondecreasing function in $[b, \infty)$.

3. If (5) has a solution $\varphi$ and $\omega_{1} \in C([b, \infty),(0, \infty))$ satisfies $0 \leq \omega_{1}(t) \leq$ $\omega(t)$ for $b \leq t<\infty$, then the equation

$$
\varphi(t)=\sigma(t)+\int_{b}^{t} K(t, s) \omega_{1}(s) g(\|\psi\|+\varphi(s)) d s
$$

has a solution $\varphi_{1}$ such that $0 \leq \varphi_{1}(t) \leq \varphi(t), b \leq t<\infty$.

Proof. 1. The necessity is clear. To prove the sufficiency we shall proceed by the method of steps. Hence we prove by mathematical induction that for each $m=1,2, \ldots$ :

(a) There exists a solution $y_{m} \in C([b, b+m],[0, \infty))$ of (5) satisfying the inequalities $0 \leq y_{m}(t) \leq \lambda(t), b \leq t \leq b+m$, and

(b) $y_{m+1}(t)=y_{m}(t), \quad b \leq t \leq b+m$.

Consider the partially ordered Banach space $X_{1}=C([b, b+1], R)$ with the sup-norm where $z_{1} \leq z_{2}$ if and only if $z_{1}(t) \leq z_{2}(t)$ for each $t \in[b, b+1]$ and each pair $z_{1}, z_{2}$ from that space. Then, by definition, the interval $\left\langle z_{1}, z_{2}\right\rangle=$ $\left\{y \in X_{1}: z_{1}(t) \leq y(t) \leq z_{2}(t), b \leq t \leq b+1\right\}$. The operator $U_{1}: X_{1} \rightarrow X_{1}$ defined by

$$
U_{1}(t)=\sigma(t)+\int_{b}^{t} K(t, s) \omega(s) g(\|\psi\|+y(s)) d s, \quad b \leq t \leq b+1,
$$

is completely continuous, nondecreasing, and, in view of (7), maps the interval $\left\langle 0,\left.\lambda\right|_{[b, b+1]}\right\rangle$ into itself. Hence by Schauder's fixed point theorem $U_{1}$ has a fixed point $Y_{1}$ satisfying $(6)$ on $[b, b+1]$. 
Suppose now that there exists a solution $y_{m}$ of $(5)$ on $[b, b+m]$. Consider the space $X_{m+1}=C([b, b+m+1], R)$ with the sup-norm and with the natural ordering. Let $U_{m+1}$ be the operator given by the right-hand side of (5) on $[b, b+m+1] . U_{m+1}$ is completely continuous, nondecreasing, and maps the interval $\left\langle 0,\left.\lambda\right|_{[b, b+m+1]}\right\rangle=\left\{y \in X_{m+1}: 0 \leq y(t) \leq \lambda(t), b \leq\right.$ $t \leq b+m+1\}$ into itself. Similarly, $U_{m+1}$ maps the closed and convex set $Y_{m+1}=\left\{x \in X_{m+1}: x(t)=y_{m}(t), b \leq t \leq b+m\right\}$ into itself. Hence $U_{m+1}\left(\left\langle 0,\left.\lambda\right|_{[b, b+m+1]}\right\rangle \cap Y_{m+1}\right) \subset\left\langle 0,\left.\lambda\right|_{[b, b+m+1]}\right\rangle \cap Y_{m+1}$ and there exists a fixed point $y_{m+1}$ of $U_{m+1}$ in $\left\langle 0,\left.\lambda\right|_{[b, b+m+1]}\right\rangle \cap Y_{m+1}$. This is the sought-for function $y_{m+1}$ with the properties (a) and (b). Then the function $\varphi(t)=$ $y_{m}(t)$ for $b \leq t \leq b+m, m=1,2, \ldots$, is a solution of $(5)$ in $[b, \infty)$, satisfying $(6)$.

2. The statement follows from (3), (4) and (5).

3. Since each solution $\varphi$ of (5) is an upper solution of (8), Statement 1 implies Statement 3.

The existence of an upper solution $\lambda$ of (5) is provided by

Lemma 4. Let $\psi, \sigma$, and $K$ have the same meaning as in Lemma 3 and let $g \in C([0, \infty),(0, \infty))$ be a nondecreasing function. Then $\lambda \in C([b, \infty,[0, \infty))$ is an upper solution of (5) if there is a function $\rho \in C([b, \infty),[0, \infty))$ such that

$$
\lambda(t)=\sigma(t)+\int_{b}^{t} K(t, s) \rho(s) d s, \quad b \leq t<\infty,
$$

and

$$
0 \leq \omega(t) \leq \frac{\rho(t)}{g(\|\psi\|+\lambda(t))}, \quad b \leq t<\infty
$$

Proof. By combining (9), (10) we get that $\lambda$ determined by (9) is an upper solution of $(5)$ in $[b, \infty)$.

Remark 1. Similarly, the necessary and sufficient condition for $\lambda \in$ $C([b, \infty,[0, \infty))$ to be a solution of $(5)$ is that there exists a function $\rho \in$ $C([b, \infty,[0, \infty))$ such that $(9)$ is fulfilled and

$$
\omega(t)=\frac{\rho(t)}{g(\|\psi\|+\lambda(t))}, \quad b \leq t<\infty
$$




\section{$\S 2$. The Main Theorem}

The main theorem reads as follows.

Theorem 1. Let $\psi \in H_{p-1}, f \in C\left([b, \infty) \times H_{p-1} \times \cdots \times h_{0}, R^{d}\right)$. Let, further, $\omega \in C([b, \infty),[0,, \infty)), g \in C([0, \infty),[0, \infty))$ be a nondecreasing function, and let

(ix) $\mid f\left(t, \chi_{t}, \ldots, \chi_{t}^{(p-1)} \mid \leq \omega(t) g\left(\left\|\chi_{t}\right\|\right)\right.$

for each $(t, \chi) \in[b, \infty) \times M^{*}$, where

$$
\begin{gathered}
M^{*}=\left\{x \in X^{*}: \sum_{k=0}^{p-1}\left|x^{(k)}(t)-\psi^{(k)}(0)\right| \leq \varphi(t) \text { for } t \geq b \quad\right. \text { and } \\
\left.x_{b}^{(k)}=\psi^{(k)}, \quad k=0, \ldots, p-1\right\}
\end{gathered}
$$

and $\varphi$ is a solution of equation (5) where the functions $\sigma$ and $K$ are determined by (3) and (4), respectively.

Then the problem $(1),(2)$ has a solution $x$ lying on $M^{*}$ and the set $F^{*}$ is a compact $R_{\delta}$-set in the space $X^{*}$.

Proof. Consider the set

$$
\begin{gathered}
M=\left\{x \in X: \sum_{k=0}^{p-1}\left|x^{(k)}(t)-\psi^{(k)}(0)\right| \leq \varphi(t) \text { for } t \geq b\right. \text { and } \\
\left.x^{(k)}(b)=\psi^{(k)}(0), \quad k=0, \ldots, p-1\right\} .
\end{gathered}
$$

Clearly, the restriction $P: X^{*} \rightarrow X$ determined by $P(x)=\left.x\right|_{[b, \infty)}$ is a homeomorphism of $M^{*}$ onto $M$. Let the map $T: m \rightarrow X$ be determined by

$$
\begin{gathered}
T(x)(t)=\sum_{k=0}^{p-1} \frac{\psi^{(k)}(0)}{k !}(t-b)^{k}+ \\
+\int_{b}^{t} \frac{(t-s)^{p-1}}{(p-1) !} f\left[s,\left(P^{-1} x\right)_{s}, \ldots,\left(P^{-1} x\right)_{s}^{(p-1)}\right] d s, \quad x \in M, \quad t \geq b,
\end{gathered}
$$

where $P^{-1}$ is the inverse of $\left.P\right|_{M^{*}}$. Then $F^{*}=P^{-1}(F)$, where $F$ is the set of all fixed points of the map $T$. Since the homeomorphic image of the compact $R_{\delta}$-set is again a compact $R_{\delta}$-set, it is sufficient to prove that $F$ is a compact $R_{\delta}$-set in the space $X$. This will be done by using Lemma 2 where 
we put $r_{k}=\psi^{(k)}(0), k=0, \ldots, p-1$. Due to (ix) the maps $T_{n}: M \rightarrow X$ determined by

$$
T_{n}(x)(t)=\left\{\begin{aligned}
& \sum_{k=0}^{p-1} \frac{\psi^{(k)}(0)}{k !}(t-b)^{k} \text { for } b \leq t \leq b+\frac{1}{n}, \\
& \sum_{k=0}^{p-1} \frac{\psi^{(k)}(0)}{k !}(t-b)^{k}+\int_{b}^{t-\frac{1}{n}} \frac{\left(t-\frac{1}{n}-s\right)^{p-1}}{(p-1) !} \times \\
& \times f\left[s,\left(P^{-1} x\right)_{s}, \ldots,\left(P^{-1} x\right)_{s}^{(p-1)}\right] d s \\
& \text { for } b+\frac{1}{n} \leq t<\infty \text { and } x \in M
\end{aligned}\right.
$$

are, together with $T$, compact and, again by (ix),

$$
\begin{gathered}
\sum_{l=0}^{p-1}\left|\left(T_{n}(x)\right)^{(l)}(t)-(T(x))^{(l)}(t)\right| \leq \\
\leq\left\{\begin{array}{l}
\sum_{l=0}^{p-1} \int_{b}^{t} \frac{(t-s)^{p-1-l}}{(p-1-l) !} \omega(s) g\left(\left\|\left(p^{-1} x\right)_{s}\right\|\right) d s, \quad b \leq t \leq b+\frac{1}{n}, \\
\sum_{l=0}^{p-1}\left\{\int_{b}^{t-\frac{1}{n}} \frac{(t-s)^{p-1-l}-\left(t-\frac{1}{n}-s\right)^{p-1-l}}{(p-1-l) !} \omega(s) g\left(\left\|\left(p^{-1} x\right)_{s}\right\|\right) d s+\right. \\
\left.+\int_{t-\frac{1}{n}}^{t} \frac{(t-s)^{p-1-l}}{(p-1-l) !} \omega(s) g\left(\left\|\left(p^{-1} x\right)_{s}\right\|\right)\right\} d s, \quad b+\frac{1}{n} \leq t<\infty .
\end{array}\right.
\end{gathered}
$$

By Lemma $3 \varphi$ is nondecreasing in $[b, \infty)$ and therefore $\left\|\left(P^{-1} x\right)_{s}\right\| \leq$ $\psi+\varphi(s)$ for each $b \leq s<\infty, x \in M$. Hence, using also (4), we get

$$
\sum_{l=0}^{p-1}\left|\left(T_{n}(x)\right)^{(l)}(t)-(T(x))^{(l)}(t)\right| \leq \varphi_{n}(t), \quad t \geq b, \quad x \in M,
$$

where

$$
\varphi_{n}(t)=\left\{\begin{array}{l}
\int_{b}^{t} K(t, s) \omega(s) g(\|\psi\|+\varphi(s)) d s, \quad b \leq t \leq b+\frac{1}{n} \\
\int_{b}^{t} K(t, s) \omega(s) g(\|\psi\|+\varphi(s)) d s-\int_{b}^{t-\frac{1}{n}} K\left(t-\frac{1}{n}, s\right) \omega(s) \times \\
\times(\|\psi\|+\varphi(s)) d s, \quad b+\frac{1}{n} \leq t<\infty, n \in N .
\end{array}\right.
$$

Clearly, $\varphi_{n} \in C([b, \infty),[0, \infty))$ and the relations $\varphi_{n+1}(t) \leq \varphi_{n}(t), \lim _{n \rightarrow \infty} \varphi_{n}(t)$ $=0$ can be proved for each $t \geq b$. Therefore these functions satisfy the assumptipons (v), (vi) of Lemma 2.

Further,

$$
\sum_{l=0}^{p-1}\left|\left(T_{n}(x)\right)^{(l)}(t)-\varphi^{(l)}(0)\right| \leq \varphi_{* n}(t), \quad t \geq b, \quad x \in M,
$$

where

$$
\varphi_{* n}=\left\{\begin{array}{l}
\sigma(t), \quad b \leq t \leq b+\frac{1}{n} \\
\sigma(t)+\int_{b}^{t-\frac{1}{n}} K\left(t-\frac{1}{n}, s\right) \omega(s) g(\|\psi\|+\varphi(s)) d s \\
b+\frac{1}{n} \leq t<\infty
\end{array}\right.
$$


$n \in N, \sigma$ and $K$ are the functions introduced by (3) and (4). The functions $\varphi_{* n}$ are nonnegative continuous functions on $[b, \infty)$ and, by virtue of $(5)$ we obtain $\varphi_{n}(t)+\varphi_{* n}(t)=\varphi(t), t \geq b, n \in N$. Hence the assumption (vii) of Lemma 2 is satisfied, too. Thus it remains for us to show that the assumption (viii) holds, and then Lemma 2 will imply the statement of Theorem 1.

Let $n \in N$ be arbitrary but fixed. If $x, y \in M, x \neq y$, then there exists a $t_{0} \in[b, \infty)$ such that $x\left(t_{0}\right) \neq y\left(t_{0}\right)$. If $b \leq t_{0} \leq b+\frac{1}{n}$, then, taking into account (12), we obtain $x\left(t_{0}\right)-T_{n}(x)\left(t_{0}\right) \neq y\left(t_{0}\right)-T_{n}(y)\left(t_{0}\right)$. In the other case there is a $t_{1} \geq b+\frac{1}{n}$ such that $t_{1}=\sup \{\tau>b: x(t)=y(t)$ for $b \leq t<$ $\tau\}$. Then there exists $t_{0} \in\left(t_{1}, t_{1}+\frac{1}{n}\right)$ such that $x\left(t_{0}\right) \neq y\left(t_{0}\right)$. By (12) we now have

$$
\begin{aligned}
& T_{n}(x)\left(t_{0}\right)=\sum_{k=0}^{p=1} \frac{\psi^{(k)}(0)}{k !}\left(t_{0}-b\right)^{k}+\int_{b}^{t_{0}-\frac{1}{n}} \frac{\left(t_{0}-\frac{1}{n}-s\right)^{p-1}}{(p-1) !} \times \\
& \times f\left[s,\left(P^{-1} x\right)_{s}, \ldots,\left(P^{-1} x\right)_{s}^{(p-1)}\right] d s=\sum_{k=0}^{p-1} \frac{\psi^{(k)}(0)}{k !}\left(t_{0}-b\right)^{k}+ \\
& +\int_{b}^{t_{0}-\frac{1}{n}} \frac{\left(t_{0}-\frac{1}{n}-s\right)^{p-1}}{(p-1) !} f\left[s,\left(P^{-1} y\right)_{s}, \ldots,\left(P^{-1} y\right)_{s}^{(p-1)}\right] d s=T_{n}(y)\left(t_{0}\right)
\end{aligned}
$$

and thus $x\left(t_{0}\right)-T_{n}(x)\left(t_{0}\right) \neq y\left(t_{0}\right)-T_{n}(y)\left(t_{0}\right)$, which we were to prove.

\section{REFERENCES}

1. J. Dugundji and A. Granas, Fixed point theory. PWN, Warszawa, 1982.

2. Z. Kubáček, Remarks on the paper of K. Czarnowski and T. Pruszko "On the structure of fixed point sets...." Preprint.

3. V. Šeda and Š. Belohorec, A remark on second-order functional differential system (submitted to Arch. Math.).

(Received 15.04.1993)

Authors' addresses:

Valter Šeda

Comenius University

Department of Mathematical Analysis

Mlynská dolina

84215 Bratislava, Slovakia
Jozef Eliaš

Slovak Technical University

Department of Mathematics

Mám. Slobody 17

81231 Bratislava, Slovakia 